\title{
CENTRALIZER NEAR-RINGS OVER FREE RING MODULES
}

\author{
C. J. MAXSON and A. P. J. VAN DER WALT
}

(Received 25 September 1989)

Communicated by B. J. Gardner

\begin{abstract}
We treat centralizer near-rings over ring modules in general, with particular emphasis on the case of free modules. Questions like the following are answered. When is the near-ring a nonring? When is the near-ring simple? What are its maximal and minimal left ideals? What is its subgroup structure? What is the radical? The cases where the ring concerned is a PID or a feld are treated in some detail.
\end{abstract}

1980 Mathematics subject classification (Amer. Math. Soc.) (1985 Revision): 16 A 76.

\section{Introduction}

Let $(G,+)$ be any group, and $A$ a set of endomorphisms of $G$. The nearring

$$
M_{A}(G):=\{f: G \rightarrow G \mid f(g \alpha)=f(g) \alpha, \text { for all } g \in G, \alpha \in A\}
$$

has been the subject of investigation in numerous papers, as is evident from a near-ring bibliography as given, for example, in [4] or [5]. One particular case has received less attention than one might have expected, viz. the case where $G$ is a right $R$-module, $R$ a ring, and $A \subseteq R$. Apart from Maxson and Smith [2], where the case of a simple artinian ring is treated and [3] where finite semisimple rings are treated, there does not seem to exist a systematic study of the interplay between the properties of a ring $R$, a right $R$-module $G$ and the near-ring $M_{R}(G)$. It is the object of this paper to lay some of the groundwork for such an investigation. In [2] it is shown that if $R$ is simple artinian, not a field, then $M_{R}(G)$ is, in fact, the left endomorphism ring of (c) 1991 Australian Mathematical Society 0263-6115/91\$A2.00+0.00 
$G_{R}$. We therefore start by considering some general conditions for $M_{R}(G)$ to be a nonring (that is, not a ring). The rest of Section 2 is devoted to some general results for the case where $G$ is a free module of finite rank, and, in fact, since it is easy to see that rank 2 shows all the salient features, we present our results only for the case $G=R^{2}$. In a number of our proofs we use the fact that $\mathrm{M}_{2}(R)$, the ring of $2 \times 2$ matrices over $R$, is a subring of $M_{R}\left(R^{2}\right)$. Indeed, this fact accounts for much of the flavour of the results.

Section 3 treats the case $M_{D}\left(D^{2}\right), D$ a principal ideal domain, in some detail. In particular, we investigate the left ideal structure with regard to both minimal and maximal left ideals. In some of this work we use ultrafilters in much the same way that they were used by Fuchs [1]. Also, the invariant subgroups of $M_{D}\left(D^{2}\right)$ are determined. The section closes with a brief discussion of the case $M_{F}\left(F^{2}\right)$, where $F$ is a field.

\section{General results}

Let $R$ be a ring with identity. All modules will be unitary. For any subset $S$ of a ring or a module, we let $S^{*}:=S \backslash\{0\}$. In this section we collect several results concerning the structure of the near-ring $M_{R}\left(R^{2}\right)$. We start by presenting conditions which imply, in many very general situations, that $M_{R}\left(R^{2}\right)$ is a near-ring, but not a ring. In fact we give our conditions for any right $R$-module $G$.

LEMMA 2.1. Let $G$ be a right $R$-module and suppose $G=H \oplus K, H$ and $K R$-submodules of $G$. Let $f \in M_{R}(G)$. If, for some non-zero $a \in H$, $b \in K, f(a+b) \neq f(a)+f(b)$ then $f$ is not a distributive element of $M_{R}(G)$.

Proof. If $e_{H}$ and $e_{K}$ are the idempotents associated with the decomposition $G=H \oplus K$, then $e_{H}, e_{K} \in M_{R}(G)$ and $e_{H}+e_{K}=$ id, the identity map in $M_{R}(G)$. Thus $f\left(e_{H}+e_{K}\right)=f$ whereas $\left(f e_{H}+f e_{K}\right)(a+b)=f(a)+f(b) \neq$ $f(a+b)$ so $f\left(e_{H}+e_{K}\right) \neq f e_{H}+f e_{K}$.

Theorem 2.2. Let $G$ be a right $R$-module with a non-trivial direct decomposition $G=H \oplus K$. If there exists a non-empty subset $X$ of $G$ such that

(i) $0 \notin X, X \neq G^{*}$,

(ii) $x \in X$ implies $x r \in X \cup\{0\}$, for all $r \in R$,

(iii) $x r \in X, r \in R$ imply $x \in X$,

then $M_{R}(G)$ is not a ring. 
Proof. Define $f: G \rightarrow G$ by

$$
f(x)= \begin{cases}x, & x \in X, \\ 0, & \text { otherwise } .\end{cases}
$$

It is straightforward, from the defining properties of $X$, to show that $f \in$ $M_{R}(G)$. We next show that there exist $a \in H, b \in K$ such that $f(a+b) \neq$ $f(a)+f(b)$. From the above lemma, $f$ is not a distributive element of $M_{R}(G)$ so $M_{R}(G)$ is not a ring.

CASE (i). $H \cap X=\varnothing, K \cap X=\varnothing$. Then for $x \in X, x=a+b$, with $a \in H, b \in K$. From this we find $f(x)=x \neq 0=f(a)+f(b)$.

Case (ii). $H \cap X \neq \varnothing, K \cap X=\varnothing$. Let $a \in H \cap X$ and choose $0 \neq b \in K$. Then

$$
f(a+b)= \begin{cases}a+b, & a+b \in X, \\ 0, & \text { otherwise },\end{cases}
$$

while $f(a)+f(b)=a$. Therefore $f(a+b) \neq f(a)+f(b)$.

CASE (iii). $H \cap X \neq \varnothing, K \cap X \neq \varnothing$. If $H \cup K \subseteq X \cup\{0\}$, then since $X \neq G^{*}$ there exists $y=a+b \in G^{*} \backslash X, a \in H, b \in K$. Hence $f(a+b)=$ $0 \neq a+b=f(a)+f(b)$. However, if (say) $H \nsubseteq X \cup\{0\}$, we take $a \in H^{*} \backslash X$ and $b \in K \cap X$. Then

$$
f(a+b)= \begin{cases}a+b, & a+b \in X, \\ 0, & \text { otherwise, }\end{cases}
$$

while $f(a)+f(b)=b$, which shows $f(a+b) \neq f(a)+f(b)$.

We next discuss a construction which will identify possible candidates for the role of $X$ in the above theorem.

Define a relation $\sim$ on $G$ by $0 \sim 0$ and for $x, y \in G^{*}, x \sim y$ if there exist $c_{i} \in G^{*}, i=1,2, \ldots, n$, with $x=c_{1}, y=c_{n}$ and $c_{i} R \cap c_{i+1} R \neq\{0\}$, $i=1,2, \ldots, n-1$. Then $\sim$ is an equivalence relation and the equivalence classes are called connected components or components of $G$. If $G^{*}$ is a component, we say $G$ is $R$-connected. We note that when $G$ is not $R$ connected the non-zero components $X$ satisfy the conditions (i), (ii), (iii) of Theorem 2.2. This gives the following result.

COROLlary 2.3. Let $G$ be a right $R$-module with a non-trivial direct decomposition. If $M_{R}(G)$ is a ring then $G$ is $R$-connected.

The converse of this corollary is not true, as is shown by $R:=\mathbb{Z}_{6}$ and $G:=\left(\mathbb{Z}_{6}\right)^{2}$. One verifies that $G$ is $R$-connected but as we see from the next result, $M_{R}(G)$ is not a ring. Recall that a proper ideal $P$ in a ring $R$ is called completely prime if $R \backslash P$ is a multiplicatively closed set. 
THEOREM 2.4. Let $R$ be a ring with a non-zero proper completely prime ideal $P$ such that $\operatorname{Ann}_{R}(P) \neq\{0\}$. Then $M_{R}\left(R^{2}\right)$ is not a ring.

Proof. Let $d \in \operatorname{Ann}_{R}(P), d \neq 0$ and let $C:=R \backslash P$, a multiplicatively closed set. Define $f: R^{2} \rightarrow R^{2}$ by

$$
f\left[\begin{array}{l}
a \\
b
\end{array}\right]= \begin{cases}{\left[\begin{array}{c}
d a \\
0
\end{array}\right],} & \text { if }\left[\begin{array}{l}
a \\
b
\end{array}\right] \in C^{2}, \\
{\left[\begin{array}{l}
0 \\
0
\end{array}\right],} & \text { otherwise. }\end{cases}
$$

To see that $f \in M_{R}\left(R^{2}\right)$ observe that

$$
\left[\begin{array}{l}
a \\
b
\end{array}\right] \in C^{2}, \quad r \in C \text { if and only if }\left[\begin{array}{l}
a r \\
b r
\end{array}\right] \in C^{2},
$$

$\left[\begin{array}{l}a \\ b\end{array}\right] \in C^{2}, \quad p \in P$ imply $\left[\begin{array}{c}d a p \\ 0\end{array}\right]=\left[\begin{array}{l}0 \\ 0\end{array}\right]=\left[\begin{array}{c}d a \\ 0\end{array}\right] p$, and

iii) $\quad\left[\begin{array}{l}a \\ b\end{array}\right] \notin C^{2}$ implies $\left[\begin{array}{l}a \\ b\end{array}\right] r \notin C^{2}, \quad$ for all $r \in R$.

Now $f \in M_{R}\left(R^{2}\right)$, and moreover,

$$
f\left(\left[\begin{array}{l}
1 \\
0
\end{array}\right]+\left[\begin{array}{l}
0 \\
1
\end{array}\right]\right)=\left[\begin{array}{l}
d \\
0
\end{array}\right] \neq\left[\begin{array}{l}
0 \\
0
\end{array}\right]
$$

while

$$
f\left[\begin{array}{l}
1 \\
0
\end{array}\right]+f\left[\begin{array}{l}
0 \\
1
\end{array}\right]=\left[\begin{array}{l}
0 \\
0
\end{array}\right] \text {. }
$$

Hence from Lemma 2.1, $f$ is not distributive so $M_{R}\left(R^{2}\right)$ is not a ring.

On the other hand, if $D$ is an integral domain, not necessarily commutative, then $M_{D}\left(D^{2}\right)$ is never a ring. For in this situation, $\left[\begin{array}{l}1 \\ 0\end{array}\right] \sim\left[\begin{array}{l}a \\ b\end{array}\right]$ if and only if $a \neq 0$ and $b=0$. Thus $X=\left\{\left[\begin{array}{l}a \\ 0\end{array}\right] \mid a \neq 0\right\}$ is a non-zero component and $X \neq D^{2}$.

COROLlARY 2.5. For every $n>1, M_{\mathbf{Z}_{n}}\left(\mathbb{Z}_{n}^{2}\right)$ is not a ring.

Proof. If $n$ is a prime, $\mathbb{Z}_{n}$ is a field and so the result follows from the above remarks. If $n=p t, p$ a prime and $t>1$, choose $P=\langle p\rangle$, the ideal generated by $p$ and let $d=t \in \operatorname{Ann}_{\mathbf{z}_{n}}(\langle p\rangle)$. Now apply Theorem 2.4.

It should be pointed out, however, that rings do arise naturally as $M_{R}(G)$. In fact, as mentioned in the introduction, if $R$ is any Artinian simple ring, not a field, and $V$ any right $R$-module then $M_{R}(V)=\operatorname{End}_{R} V$ is a ring. Further, if $G$ is a cyclic $R$-module then $M_{R}(G)$ is a ring. As a specific 
instance of this case let $G$ be a finitely generated abelian group and let $R$ denote the endomorphism ring of $G$. Then $M_{R}(G)$ is a ring. As a final example here, we let $D$ be a commutative integral domain with field of quotients $Q(D)$. Then $M_{D}(Q(D))$ is a ring.

We next turn to a consideration of the structure of the near-ring $M_{R}\left(R^{2}\right)$. We first establish a very useful connection between this near-ring and the subring $\mathbf{M}_{2}(R)$.

LEMMA 2.6. Let $f \in M_{R}\left(R^{2}\right)$ and let $A$ be a matrix with at most one non-zero column in $\mathrm{M}_{2}(R)$, say

$$
A:=\left[\begin{array}{ll}
0 & b_{1} \\
0 & b_{2}
\end{array}\right]
$$

Then

$$
f \circ A=\left[\begin{array}{ll}
0 & c_{1} \\
0 & c_{2}
\end{array}\right], \quad \text { where } f\left[\begin{array}{l}
b_{1} \\
b_{2}
\end{array}\right]=\left[\begin{array}{l}
c_{1} \\
c_{2}
\end{array}\right]
$$

Proof. let $\left[\begin{array}{l}x \\ y\end{array}\right]$ be arbitrary in $R^{2}$. We have $f \circ\left[\begin{array}{ll}0 & b_{1} \\ 0 & b_{2}\end{array}\right]\left[\begin{array}{l}x \\ y\end{array}\right]=f\left[\begin{array}{l}b_{1} y \\ b_{2} y\end{array}\right]=\left(f\left[\begin{array}{l}b_{1} \\ b_{2}\end{array}\right]\right) y=\left[\begin{array}{l}c_{1} \\ c_{2}\end{array}\right] y=\left[\begin{array}{l}c_{1} y \\ c_{2} y\end{array}\right]=\left[\begin{array}{ll}0 & c_{1} \\ 0 & c_{2}\end{array}\right]\left[\begin{array}{l}x \\ y\end{array}\right]$, and hence we have the result.

CoRollary 2.7. If $C_{k}$ denotes the left ideal of $\mathrm{M}_{2}(R)$ consisting of matrices which are zero except possibly in the kth column, then $C_{k}$ is a (left) $M_{R}\left(R^{2}\right)$-subgroup.

THEOREM 2.8. If $R$ is a simple ring then $M_{R}\left(R^{2}\right)$ is a simple near-ring.

Proof. Let $H$ be a non-zero ideal of $M_{R}\left(R^{2}\right)$ and let $f \in H, f \neq 0$. Thus there exists $\left[\begin{array}{l}x \\ y\end{array}\right] \neq\left[\begin{array}{l}0 \\ 0\end{array}\right]$ in $R^{2}$ such that $f\left[\begin{array}{l}x \\ y\end{array}\right] \neq\left[\begin{array}{l}0 \\ 0\end{array}\right]$. Therefore by Lemma 2.6,

$$
f\left[\begin{array}{ll}
x & 0 \\
y & 0
\end{array}\right] \neq\left[\begin{array}{ll}
0 & 0 \\
0 & 0
\end{array}\right] \text { and } f\left[\begin{array}{ll}
x & 0 \\
y & 0
\end{array}\right] \in H \cap \mathbb{M}_{2}(R) .
$$

But this means that $H \cap \mathbb{M}_{2}(R)$ is a non-zero ideal in $\mathbb{M}_{2}(R)$. Since $R$ is simple, because of the correspondence between ideals in $R$ and those in $\mathbf{M}_{2}(R)$, we know $\mathbf{M}_{2}(R)$ is simple. Hence $H \cap \mathbb{M}_{2}(R)=\mathbb{M}_{2}(R)$, and consequently id $\in H$ which means $H=M_{R}\left(R^{2}\right)$.

Actually, we have established a somewhat stronger result. 
COROLLARY 2.9. If $R$ is a simple ring then $M_{R}\left(R^{2}\right)$ contains no non-trivial two-sided $M_{R}\left(R^{2}\right)$-subgroups.

We can also make use of Lemma 2.6 to show that the distributive elements in $M_{R}\left(R^{2}\right)$ are precisely the matrices in $\mathrm{M}_{R}(R)$.

THEOREM 2.10. The subnear-ring of $M_{R}\left(R^{2}\right)$ generated by the distributive elements of $M_{R}\left(R^{2}\right)$ is $\mathbf{M}_{2}(R)$.

Proof. Let $D$ denote the semigroup of distributive elements in $M_{R}\left(R^{2}\right)$. Then $\operatorname{dg} D$ is the subnear-ring of $M_{R}\left(R^{2}\right)$ distributively generated by $D$. Clearly $\mathrm{M}_{2}(R) \subseteq \operatorname{dg} D$. For the reverse inclusion let $f \in D$. Then

$$
\begin{aligned}
f & =f \circ \mathrm{id}=f \circ\left[\begin{array}{ll}
1 & 0 \\
0 & 1
\end{array}\right]=f\left(\left[\begin{array}{ll}
1 & 0 \\
0 & 0
\end{array}\right]+\left[\begin{array}{ll}
0 & 0 \\
0 & 1
\end{array}\right]\right) \\
& =f\left[\begin{array}{ll}
1 & 0 \\
0 & 0
\end{array}\right]+f\left[\begin{array}{ll}
0 & 0 \\
0 & 1
\end{array}\right] \in \mathbf{M}_{2}(R) .
\end{aligned}
$$

Thus $D \subseteq \mathbb{M}_{2}(R)$ so $\operatorname{dg} D=\mathbf{M}_{2}(R)$.

We showed above that $M_{R}\left(R^{2}\right)$ is simple when $R$ is simple. We investigate further when $M_{R}\left(R^{2}\right)$ is simple. Recall that a ring $R$ is a prime ring if for each pair $A, B$ of non-zero ideals of $R, A B \neq\{0\}$.

THEOREM 2.11. If $M_{R}\left(R^{2}\right)$ is a simple near-ring then $R$ is a prime ring.

Proof. We assume $R$ is not prime and let $A, B$ be non-zero ideals such that $A B=\{0\}$. Let $0 \neq b \in B$ and set $C:=R b \subseteq B$. We let $C^{2}=\left\{\left[\begin{array}{l}a \\ c\end{array}\right] \in\right.$ $\left.R^{2} \mid a, c \in C\right\}$ and note that $C^{2}$ is an $M_{R}\left(R^{2}\right)$-subgroup. In fact, if

$$
\left[\begin{array}{l}
r_{1} b \\
r_{2} b
\end{array}\right] \in C^{2}
$$

then for each $f \in M_{R}\left(R^{2}\right)$,

$$
f\left[\begin{array}{l}
r_{1} b \\
r_{2} b
\end{array}\right]=f\left[\begin{array}{l}
r_{1} \\
r_{2}
\end{array}\right] b=\left[\begin{array}{l}
s \\
t
\end{array}\right] b=\left[\begin{array}{l}
s b \\
t b
\end{array}\right] \in C^{2}, \quad \text { where }\left[\begin{array}{l}
s \\
t
\end{array}\right]:=f\left[\begin{array}{l}
r_{1} \\
r_{2}
\end{array}\right] .
$$

Therefore $K=\left(0: C^{2}\right)$ is a proper ideal in $M_{R}\left(R^{2}\right)$. However $K \neq\{0\}$ because if $a \in A, a \neq 0$ then $\left[\begin{array}{ll}a & 0 \\ 0 & 0\end{array}\right] \in K$. This contradiction implies that $R$ must be a prime ring.

As a partial converse we have the following. 
THEOREM 2.12. Let $R$ be an integral domain, not necessarily commutative. Then $M_{R}\left(R^{2}\right)$ is a simple near-ring.

Proof. Suppose $M_{R}\left(R^{2}\right)$ has a non-trivial ideal $J$. Let $I:=J \cap \mathbb{M}_{2}(R)$. Since $J \neq\{0\}$, as in Lemma 2.6, if $0 \neq f \in J$, say $f\left[\begin{array}{l}x \\ y\end{array}\right]=\left[\begin{array}{l}a \\ b\end{array}\right] \neq\left[\begin{array}{l}0 \\ 0\end{array}\right]$, then $f\left[\begin{array}{ll}x & 0 \\ y & 0\end{array}\right]=\left[\begin{array}{ll}a & 0 \\ b & 0\end{array}\right] \in I$, so $I \neq\{0\}$. Thus, as is well known, there exists an ideal $T$ of $R$ such that $I=M_{2}(T)$. Let $0 \neq t \in T$. Then $t-1 \notin T$, where 1 is the identity of $R$, otherwise id $\in I \subseteq J$, but $J \neq M_{R}\left(R^{2}\right)$. We shall show $\left[\begin{array}{cc}t-1 & 0 \\ 0 & 0\end{array}\right] \in J$ and hence $\left[\begin{array}{cc}t-1 & 0 \\ 0 & 0\end{array}\right] \in I$ which is a contradiction to $I=\mathbb{M}_{2}(T)$ and so $M_{R}\left(R^{2}\right)$ must be simple. To this end, since $\left[\begin{array}{ll}t & 0 \\ 0 & 0\end{array}\right] \in J$, we must have $g:=f\left(\left[\begin{array}{ll}t & 0 \\ 0 & 0\end{array}\right]+\left[\begin{array}{ll}0 & 0 \\ 1 & 0\end{array}\right]\right)-f\left[\begin{array}{ll}0 & 0 \\ 1 & 0\end{array}\right] \in J$, for all $f \in M_{R}\left(R^{2}\right)$. We construct an $f$ such that $g=\left[\begin{array}{cc}t-1 & 0 \\ 0 & 0\end{array}\right]$. Since $R$ is an integral domain, $\left[\begin{array}{l}0 \\ 1\end{array}\right]$ and $\left[\begin{array}{l}t \\ 1\end{array}\right]$ are in different connected components. (See the remark after Theorem 2.4.) Let $f: R^{2} \rightarrow R^{2}$ be defined by

$$
f\left[\begin{array}{l}
a \\
b
\end{array}\right]= \begin{cases}{\left[\begin{array}{l}
b \\
b
\end{array}\right],} & \text { if } a=0, \\
{\left[\begin{array}{l}
a \\
b
\end{array}\right],} & \text { if } a \neq 0 .\end{cases}
$$

It is straightforward to verify that $f \in M_{R}\left(R^{2}\right)$. Using this $f$, for any $\left[\begin{array}{l}x \\ y\end{array}\right] \in R^{2}$, we have

$$
\begin{aligned}
g\left[\begin{array}{l}
x \\
y
\end{array}\right] & =\left(f\left(\left[\begin{array}{ll}
t & 0 \\
0 & 0
\end{array}\right]+\left[\begin{array}{ll}
0 & 0 \\
1 & 0
\end{array}\right]\right)-f\left[\begin{array}{ll}
0 & 0 \\
1 & 0
\end{array}\right]\right)\left[\begin{array}{l}
x \\
y
\end{array}\right] \\
& =f\left(\left[\begin{array}{l}
t x \\
x
\end{array}\right]\right)-f\left[\begin{array}{l}
0 \\
x
\end{array}\right]=f\left[\begin{array}{l}
t \\
1
\end{array}\right] x-\left[\begin{array}{l}
x \\
x
\end{array}\right]=\left[\begin{array}{l}
t \\
1
\end{array}\right] x-\left[\begin{array}{l}
1 \\
1
\end{array}\right] x \\
& =\left[\begin{array}{c}
t-1 \\
0
\end{array}\right] x=\left[\begin{array}{cc}
t-1 & 0 \\
0 & 0
\end{array}\right]\left[\begin{array}{l}
x \\
y
\end{array}\right] .
\end{aligned}
$$

Thus $g=\left[\begin{array}{cc}t-1 & 0 \\ 0 & 0\end{array}\right]$, as required.

Recall that a commutative ring $R$ is prime if and only if $R$ is an integral domain. Thus from the above two theorems we have a nice characterization for $M_{R}\left(R^{2}\right)$ to be simple when $R$ is commutative.

COROLlARY 2.13. Let $R$ be a commutative ring. Then $M_{R}\left(R^{2}\right)$ is a simple near-ring if and only if $R$ is an integral domain.

We next turn to the question of when $M_{R}\left(R^{2}\right)$ is 2-semisimple, that is, when $J_{2}\left(M_{R}\left(R^{2}\right)\right)=\{0\}$. For left artinian rings $R$ we find a very tight connection between the structures of $R$ and $M_{R}\left(R^{2}\right)$. 
THEOREM 2.14. Let $R$ be a left artinian ring and let $N:=M_{R}\left(R^{2}\right)$. Then $J_{2}(N)=\{0\}$ if and only if $J(R)=\{0\}$.

Proof. Suppose $J(R) \neq\{0\}$. Choose $0 \neq u \in J(R)$, let $U:=R u$ and let $A:=\left\{\left[\begin{array}{ll}x & 0 \\ y & 0\end{array}\right] \mid x, y \in U\right\}$. Clearly $A$ is a subgroup of $N$. We show $A$ is an $N$-subgroup. Let $f \in N,\left[\begin{array}{ll}x & 0 \\ y & 0\end{array}\right] \in A$. Then there exist $r_{1}, r_{2} \in R$ such that $x=r_{1} u, y=r_{2} u$. If

$$
f\left[\begin{array}{l}
r_{1} \\
r_{2}
\end{array}\right]=\left[\begin{array}{l}
c \\
d
\end{array}\right]
$$

then for any $\left[\begin{array}{l}a \\ b\end{array}\right] \in R^{2}$,

$$
f\left[\begin{array}{l}
r_{1} u a \\
r_{2} u a
\end{array}\right]=\left[\begin{array}{l}
c \\
d
\end{array}\right] u a=\left[\begin{array}{ll}
c u & 0 \\
d u & 0
\end{array}\right]\left[\begin{array}{l}
a \\
b
\end{array}\right] .
$$

Hence $f\left[\begin{array}{ll}x & 0 \\ y & 0\end{array}\right]=\left[\begin{array}{cc}c u & 0 \\ d u & 0\end{array}\right] \in A$. Since $R$ is left artinian, $J(R)$ is nilpotent, say $(J(R))^{n}=\{0\}$. But then for $\left[\begin{array}{ll}x & 0 \\ y & 0\end{array}\right] \in A, x, y \in J(R)$ so $\left[\begin{array}{ll}x & 0 \\ y & 0\end{array}\right]^{n}=\left[\begin{array}{ll}0 & 0 \\ 0 & 0\end{array}\right]$. Therefore $A$ is a nil $N$-subgroup of $N$ and so $A \subseteq J_{2}(N)$. Hence $J_{2}(N) \neq$ $\{0\}$.

Conversely let $J(R)=\{0\}$ and let $L$ be any minimal left ideal of $R$. Thus, for all $c \in L, c \neq 0$, we have $R c=L$. Consider $L^{2} \subseteq R^{2}$. As above, if $\left[\begin{array}{l}x \\ y\end{array}\right] \in L^{2}$ then for each $f \in N, f\left[\begin{array}{l}x \\ y\end{array}\right] \in L^{2}$ so $L^{2}$ is an $N$-group. Further, since $R c=L, L^{2}$ is monogenic by $\left[\begin{array}{l}c \\ 0\end{array}\right]$ (in fact using only elements from $\left.\mathrm{M}_{2}(R)\right)$ and if $\{0\} \neq H$ is any $N$-subgroup of $L^{2}$ and $\left[\begin{array}{l}a \\ b\end{array}\right] \in H,\left[\begin{array}{l}a \\ b\end{array}\right] \neq\left[\begin{array}{l}0 \\ 0\end{array}\right]$, then $N\left[\begin{array}{l}a \\ b\end{array}\right]=L^{2}$. Thus $L^{2}$ is a type $2 N$-group. We have shown that for any minimal left ideal $L$ of $R, L^{2}$ is a type $2 N$-group.

Assume $J_{2}(N) \neq\{0\}$. From Lemma 2.6 we get $J_{2}(N) \cap M_{2}(R) \neq\{0\}$. Thus $J_{2}(N) \cap \mathbb{M}_{2}(R)=\mathbb{M}_{2}(T)$, for some non-zero ideal $T$ of $R$. Since $R$ is left artinian, $T$ contains a minimal left ideal of $E$, say $L_{0}$. Thus, for each $a \in L_{0},\left[\begin{array}{ll}a & 0 \\ 0 & 0\end{array}\right] \in \mathbb{M}_{2}(T) \subseteq J_{2}(N)$. Let $b \in L_{0}$. Then $\left[\begin{array}{ll}a & 0 \\ 0 & 0\end{array}\right]\left[\begin{array}{l}b \\ 0\end{array}\right]=\left[\begin{array}{l}0 \\ 0\end{array}\right]$ since $\mathbb{M}_{2}(T)$ annihilates every type $2 N$-group, in particular $L_{0}^{2}$. But this implies $L_{0} \cdot L_{0}=\{0\}$ which means that $L_{0}$ is a nilpotent left ideal of $R$ and as such, $L_{0} \subseteq J(R)$, contradicting the fact that $J(R)=\{0\}$. Thus we must have $J_{2}(N)=\{0\}$.

Corollary 2.15. Let $n$ be a positive integer, $n>1$ and let $N:=$ $M_{\mathbf{z}_{n}}\left(\mathbb{Z}_{n}^{2}\right)$. The following are equivalent:

(a) $N$ is 2-semisimple;

(b) $J\left(\mathbb{Z}_{n}\right)=\{0\}$;

(c) $n$ is square-free.

Proof. As is well known, $J\left(\mathbb{Z}_{n}\right)=\{0\}$ if and only if $n$ is square-free. 
We have found several connections among the structures $R, \mathrm{M}_{R}(R)$ and $M_{R}\left(R^{2}\right)$. In the next section we take $R$ to be a principal ideal domain (PID) and determine various substructures of $M_{R}\left(R^{2}\right)$.

\section{3. $M_{D}\left(D^{2}\right), D$ a PID}

In this section we investigate the internal structure of the near-ring $M_{D}\left(D^{2}\right)$. We focus primarily on the case where $D$ is a principal ideal domain (PID); however some of our results hold for more general integral domains.

To begin, we let $D$ be an integral domain, not necessarily commutative. As above we consider only the case $D^{2}$ since the general situation, $D^{n}$, $n>2$, is essentially the same. We say an element $\alpha \in D^{2}$ is a generator in $D^{2}$ if, for any $\beta \in D^{2}, \alpha r=\beta s \neq 0$ implies $\beta=\alpha t$ where $r, s, t \in D$. This is equivalent to $\alpha D \cap \beta D \neq\{0\}$ implies $\beta D \subseteq \alpha D$.

EXAMPLE 3.1. The following are generators in $D^{2}$ :
(1) $\left[\begin{array}{l}d \\ 1\end{array}\right]$;
(2) $\left[\begin{array}{l}1 \\ d\end{array}\right]$;
(3) $\left[\begin{array}{c}d+1 \\ d\end{array}\right]$, for each $d \in D$.

For, if $d=0$ the result is clear. Let $d \neq 0$ and suppose $\left[\begin{array}{l}d \\ 1\end{array}\right]=\left[\begin{array}{l}x \\ y\end{array}\right] s \neq\left[\begin{array}{l}0 \\ 0\end{array}\right]$. Then $d r=x s, r=y s$ so $d y s=x s$. But then $d y=x$ so $\left[\begin{array}{l}x \\ y\end{array}\right]=\left[\begin{array}{l}d \\ 1\end{array}\right] y$. The situation for (2) is similar. For (3) suppose $\left[\begin{array}{c}d+1 \\ d\end{array}\right] r=\left[\begin{array}{l}x \\ y\end{array}\right] s$. Then $d r+r=x s, d r=y s$ and so $y s+r=x s$, that is, $r=(x-y) s$. Consequently, $(d+1)(x-t) s=x s$ and $d(x-y) s=y s$. Hence, $\left[\begin{array}{c}d+1 \\ d\end{array}\right](x-y)=\left[\begin{array}{l}x \\ y\end{array}\right]$.

Let $S \subseteq D^{2}$. We say $S$ is a set of generators in $D^{2}$ if each $\alpha \in S$ is a generator and for $\alpha, \beta \in S, \alpha D \subseteq \beta D$ implies $\alpha=\beta$. Using sets of generators, one can construct functions in $M_{D}\left(D^{2}\right)$. In fact, suppose $S$ is a set of generators in $D^{2}, T$ is any subset of $D^{2}$ and $\varphi: S \rightarrow T$ is any function. Then it is straightforward to verify that the function $f: D^{2} \rightarrow D^{2}$ defined by

$$
f(\gamma)= \begin{cases}\varphi(\alpha) r, & \text { if } \gamma=\alpha r \text { for some } \alpha \in S, r \in D, \\ 0, & \text { otherwise, }\end{cases}
$$

is an element of $M_{D}\left(D^{2}\right)$. Thus functions in $M_{D}\left(D^{2}\right)$ can be defined by specifying their action on a set of generators. We shall use this frequently in the sequel.

Now let $D$ be a unique factorization domain (UFD), let $\sim$ be the equivalence relation defined after Theorem 2.2 and let $E$ denote an equivalence class of $\sim, E \neq\{0\}$. We show that $E$ contains a generator. 
Let $\left[\begin{array}{l}0 \\ 0\end{array}\right] \neq\left[\begin{array}{l}a \\ b\end{array}\right] \in E$ and let $d=\operatorname{gcd}(a, b)$ where $\operatorname{gcd}(x, 0)=x$. Let $d a^{\prime}=a, d b^{\prime}=b$. Since $\left[\begin{array}{c}a^{\prime} \\ b^{\prime}\end{array}\right] d=\left[\begin{array}{l}a \\ b\end{array}\right] \neq\left[\begin{array}{l}0 \\ 0\end{array}\right],\left[\begin{array}{c}a^{\prime} \\ b^{\prime}\end{array}\right] \in E$. Suppose $\left[\begin{array}{c}a^{\prime} \\ b^{\prime}\end{array}\right] r=$ $\left[\begin{array}{l}x \\ y\end{array}\right] s \neq\left[\begin{array}{l}0 \\ 0\end{array}\right]$ where without loss of generality we take $\operatorname{gcd}(r, s)$ to be a unit. We have $a^{\prime} r=x s$ and $b^{\prime} r=y s$. If $\operatorname{gcd}\left(a^{\prime}, s\right)=d^{\prime}$ is not a unit then from $b^{\prime} r=y s$ we get $d^{\prime} \mid b^{\prime}$. But this is a contradiction since $\operatorname{gcd}\left(a^{\prime}, b^{\prime}\right)$ is a unit. Thus $\operatorname{gcd}\left(a^{\prime}, s\right)$ is a unit which means $a^{\prime} \mid x$ and in the same manner, $b^{\prime} \mid y$ say $x=a^{\prime} t_{1}, y=b^{\prime} t_{2}$. But then $a^{\prime} r=x s$ implies $a^{\prime} r=a^{\prime} t_{1} s$ so $r=t_{1} s$. In a like manner we get $r=t_{2} s$, and hence $t_{1}=t_{2}$. But then $\left[\begin{array}{l}x \\ y\end{array}\right]=\left[\begin{array}{l}a^{\prime} \\ b^{\prime}\end{array}\right] t_{1}$, and hence $\left[\begin{array}{c}a^{\prime} \\ b^{\prime}\end{array}\right]$ is a generator for $E$.

THEOREM 3. Let $D$ be a UFD and let $\sim$ be the equivalence relation defined previously. Then every non-zero equivalence class $E$ has a generator, unique up to units. Further for each $0 \neq \alpha \in D^{2}$, there exists a generator $\beta$, unique $u p$ to units such that $\alpha=\beta r$ for some $r \in D$.

Proof. The existence of a generator has been established above. For uniqueness, suppose $\alpha, \beta \in D^{2}$ are generators contained in $E$. Since $D$ is a UFD and $\alpha \sim \beta$, there exist $r, s \in D$ such that $\alpha r=\beta s \neq 0$. Hence, since $\alpha, \beta$ are generators, $\alpha=\beta t_{1}, \beta=\alpha t_{2}, t_{1} t_{2} \in D$. From this we find $t_{1}$ and $t_{2}$ are units in $D$. The final remark is immediate since for each $0 \neq \alpha \in D^{2}, \alpha$ is in some equivalence class and so there exists a generator $\beta$ such that $\alpha=\beta r, r \in D$.

We next determine the minimal left ideals of $M_{D}\left(D^{2}\right), D$ a UFD. For each equivalence class $E$ there is a generator $\alpha$, unique up to units, such that the function $e_{\alpha}: D^{2} \rightarrow D^{2}$ defined by

$$
e_{\alpha}(x)= \begin{cases}x, & x \in E, \\ 0, & \text { otherwise },\end{cases}
$$

is an idempotent function in $M_{D}\left(D^{2}\right)$.

LEMMA 3.3. Every non-zero left ideal $L$ of $N:=M_{D}\left(D^{2}\right)$ contains an idempotent of the form $e_{\alpha}$ for some equivalence class $E$ with generator $\alpha$.

Proof. Let $0 \neq l \in L$ with (say) $l \neq 0$ on $E$. Then $l(\alpha) \neq 0$ so $l(\alpha)=\beta r$ for some generator $\beta$ and $r \in D$. The function $l^{\prime}: D^{2} \rightarrow D^{2}$ determined by $l^{\prime}(\beta r)=\alpha r$ for $\beta r$ in the equivalence class with generator $\beta$ and $l^{\prime}(x)=0$, otherwise, is in $N$, thus $l^{\prime} l \in N$. Moreover $l^{\prime} l$ has the property $l^{\prime} l\left(D^{2}\right) \subseteq \alpha D$. Thus we assume without loss of generality that $l$ has this property. Define $g \in N$ by $g(\alpha):=0$ and $g(x):=x, x \notin E$. Then $h:=e_{\alpha}(l+g)-e_{\alpha} g$ is in $L$ with $h(\alpha)=l(\alpha)$ and $h(x)=e_{\alpha}(l(x)+x)$, 
$x \notin E$. Since $l(x)+x \notin E, h(x)=0, x \notin E$. Define $\hat{g} \in N$ by $\hat{g}(\alpha):=\gamma$ for some generator $\gamma \neq \alpha$ and $\hat{g}(x):=0, x \notin E$. Further, define $f \in N$ by $f(\gamma):=\alpha$ and $f(x):=0, x$ not in the equivalence class determined by $\gamma$. Now consider $k:=f(h+\hat{g})-f \hat{g}$ which is in $L$ since $h \in L$. We have $k(\alpha)=f(l(\alpha)+\gamma)-f(\gamma)=-\alpha$ since $l(\alpha)+\gamma \notin E$ and $k(x)=f(0+0)-f(0)=0,0, x \notin E$. But then $-k=e_{\alpha} \in L$ as was to be shown.

COROLlaRY 3.4. If $L$ is a minimal left ideal for $N:=M_{D}\left(D^{2}\right), D a$ $U F D$, then $L=N e_{\alpha}$ for some generator $\alpha$.

Proof. If $e_{\alpha} \in L$ and $k:=f\left(g e_{\alpha}+h\right)-f h$ for $f, g, h \in N$, then $k e_{\alpha}=k$. So $N e_{\alpha}$ is a non-zero left ideal contained in $L$.

Theorem 3.5. Let $D$ be a UFD and let $N:=M_{D}\left(D^{2}\right)$. Then $N$ contains minimal left ideals and all minimal left ideals are of the form $N e_{\alpha}, \alpha$ a generator.

Proof. We only need to show that $N$ contains minimal left ideals since the rest of the statement follows from the above corollary. We know $N e_{\alpha}$ is a non-zero left ideal for each generator $\alpha$. Suppose $K$ is a non-zero left ideal contained in $N e_{\alpha}$. Then $K=N e_{\beta}$ for some generator $\beta$, and hence $e_{\beta}=f e_{\alpha}$ for some $f \in N$. But this is possible if and only if $\alpha=\beta$.

For maximal left ideals we do not have a complete characterization. However, in several situations we can identify maximal left ideals.

THeOREM 3.6. Let $M$ be a maximal left ideal of $N:=M_{D}\left(D^{2}\right), D$ a UFD. If there exists a generator $\alpha$ such that $e_{\alpha} \notin M$ then $M=\operatorname{Ann}(E)$ where $E$ is the equivalence class containing $\alpha$.

Proof. Since $e_{\alpha} \notin M, M+N e_{\alpha}=N$ which implies there exist $m \in$ $M, n \in N$, with $m+n e_{\alpha}=$ id. Thus, for $y \notin E, m(y)=y$ while for $y \in E, m(y)+n(y)=y$. Let $k:=e_{\alpha}\left(m+e_{\alpha}\right)-e_{\alpha} e_{\alpha} \in M$. Thus $k(y)=0$ for $y \notin E$ and $k(y)=e_{\alpha}(m(y)+y)-y$ for $y \in E$. Suppose $m(y) \neq 0$ for some $y \in E$. If $m(y) \in E$, then $m(y)+y \in E$, and hence in this case, $k(y)=m(y)$ which in turn gives $k(\alpha)=m(\alpha)=\alpha r$ for some $r \in D$. If $m(y) \notin E, m(y)+y \notin E$ so $k(\alpha)=-\alpha$. Thus $k$ is a non-zero element in $M$ with the property $k\left(D^{2}\right) \subseteq \alpha D$, and as in Lemma 3.3, we obtain $e_{\alpha} \in M$, a contradiction. Consequently, $m(y)=0$ for all $y \in E$. Now let $h$ be an arbitrary in $\operatorname{Ann}(E)$ and let $l:=h\left(m+e_{\alpha}\right) k-h e_{\alpha} \in M$. For $y \in E, l(y)=0$; for $y \notin E, l(y)=h(m(y))=h(y)$, that is, $l=h$. This 
shows $\operatorname{Ann}(E) \subseteq M$. But from $N / \operatorname{Ann}(E) \cong N e_{\alpha}$ and the fact that $N e_{\alpha}$ is a minimal left ideal we get $\operatorname{Ann}(E)$ is a maximal left ideal, and hence $M=\operatorname{Ann}(E)$.

From the above proof we obtain the following.

Corollary 3.7. Let $D$ be a UFD, $N:=M_{D}\left(D^{2}\right)$ and let $M$ be a maximal left ideal of $N$. Then $M=\operatorname{Ann}(E)$ for some equivalence class $E$, or for each generator $\alpha, e_{\alpha} \in M$.

When $D$ is finite and thus a field, the equivalence classes are the orbits of the action of $D^{*}$ on $D^{2}$. In this case we obtain the known result [5, 9.214] that the maximal left ideals are precisely the annihilators of single orbits. On the other hand, when $D$ is infinite and $M$ is a maximal ideal which is not an annihilator of an equivalence class then $M \supseteq \sum \bigoplus N e_{\alpha}$ where $\alpha$ ranges over a set of generators, one from each equivalence class. As the next example points out, $\sum \bigoplus N e_{\alpha}$ is in general not a maximal left ideal.

EXAMPLE 3.8. Let $D:=\mathbb{Z}, N:=M_{D}\left(D^{2}\right), H$ a set of generators, one chosen from each equivalence class. Further let $L:=\sum_{\alpha \in H} \oplus N e_{\alpha}$ and consider the subset $\left\{\left[\begin{array}{c}1 \\ m\end{array}\right] \mid m \in \mathbb{Z}^{+}\right\}$of $H$. Define

$$
T:=\left\{f \in N \mid \text { for some positive integer } k, f\left(\left[\begin{array}{l}
1 \\
m
\end{array}\right]\right)=\left[\begin{array}{l}
0 \\
0
\end{array}\right], m>k\right\} \text {. }
$$

One verifies that $T$ is a left ideal of $N$, and $L \subseteq T$ since $e_{\alpha} \in T$ for all $\alpha \in H$. But $L \neq T$, for if $f$ is the function such that $f\left[\begin{array}{l}1 \\ m\end{array}\right]=\left[\begin{array}{l}0 \\ 0\end{array}\right]$ for all $m \in \mathbb{Z}^{+}$but $f\left[\begin{array}{l}a \\ b\end{array}\right]=\left[\begin{array}{l}a \\ b\end{array}\right]$ for all generators $\left[\begin{array}{l}a \\ b\end{array}\right] \in H \backslash\left\{\left[\begin{array}{l}1 \\ m\end{array}\right] \mid m \in \mathbb{Z}^{+}\right\}$then $f \notin L$.

We now turn to another method for obtaining maximal left ideals. For this method we only require that $D$ be an integral domain, not necessarily commutative and not a field. Again we let $N:=M_{D}\left(D^{2}\right)$ and let $H$ denote a collection of distinct generators in $D^{2}$ where two generators $\alpha, \beta$ are distinct if $\alpha D \cap \beta D=\{0\}$. Let $\mathscr{F}$ be an ultrafilter on $H$. That is, $\mathscr{F}$ is a non-empty collection of subsets of $H$ with the properties (i) $\varnothing \notin \mathscr{F}$, (ii) if $A, B \in \mathscr{F}$ then $A \cap B \in \mathscr{F}$, (iii) if $A \in \mathscr{F}$ and $A \subseteq B$ then $B \in \mathscr{F}$, and (iv) for each $A \subseteq H$, either $A \in \mathscr{F}$ or $H \backslash A \in \mathscr{F}$. Define $L(\mathscr{F}):=\{f \in N \mid f(A)=\{0\}$ for some $A \in \mathscr{F}\}$. It is straightforward to verify that $L(\mathscr{F})$ is a left ideal of $N$. If $\mathscr{F}$ is the principal filter generated by a single generator, say $\alpha$, then we note that $L(\mathscr{F})=\operatorname{Ann}\{\alpha\}$. Of course when $D$ is a UFD this agrees with $\operatorname{Ann}(E)$ where $D$ is the equivalence class with generator $\alpha$. We now show that if $H$ is countable, or more generally if every $A \in \mathscr{F}$ contains a countable subset which is also in $\mathscr{F}$, then $L(\mathscr{F})$ is a maximal left ideal of $N$. 
To this end, let $f \notin L(\mathscr{F})$ and let $Z_{f}=\{x \in D \mid f(x)=0\}$. Since $f \notin L(\mathscr{F}), Z_{f} \notin \mathscr{F}$ so we have $T:=H \backslash Z_{f} \in \mathscr{F}$ and $f$ is non-zero on $T$. Let $S$ be a countable subset of $T$ with $S \in \mathscr{F}$. Let $g: D^{2} \rightarrow D^{2}$ be defined by $g(\alpha)=0, \alpha \in S, g(\alpha)=f(\alpha), \alpha \notin S$. Then $g \in N$ and in fact $g \in L(\mathscr{F})$. Consequently $f-g \notin L(\mathscr{F})$ and $(f-g)(\alpha)=f(\alpha)$, $\alpha \in S$, while $(f-g)(\alpha)=0, \alpha \notin S$. So, without loss of generality we take $f$ to be non-zero on $S$ and zero off $S, S=\left\{\sigma_{i}\right\}$, a countable set of generators.

Let $f^{\prime}$ be the function in $N$ defined by

$$
f^{\prime}\left[\begin{array}{l}
a \\
b
\end{array}\right] k= \begin{cases}{\left[\begin{array}{l}
a \\
a
\end{array}\right]} & \text { if } j \neq 0, \\
{\left[\begin{array}{l}
b \\
b
\end{array}\right]} & \text { if } a=0,\end{cases}
$$

where $\left[\begin{array}{l}a \\ b\end{array}\right] \in D^{2}$. Then $f^{\prime} f$ is a function which is zero off $S$, non-zero on $S$ with the $f^{\prime} f(\alpha)=\left[\begin{array}{l}x \\ x\end{array}\right]$ for some $x=x(\alpha)$, all $\alpha \in S$. We wish to construct a function $l \in N$ in the left ideal generated by $f$ which will be the identity on $S$. We will then show that the ideal generated by $L(F) \cup\{f\}$ is all of $N$ which means that $L(\mathscr{F})$ is a maximal left ideal. Now $f^{\prime} f$ is in the left ideal generated by $f$ so again without loss of generality we call this function $f$. We have therefore

$$
\begin{aligned}
f\left(\sigma_{i}\right) & =\left[\begin{array}{l}
x_{i} \\
x_{i}
\end{array}\right] \neq\left[\begin{array}{l}
0 \\
0
\end{array}\right], \quad \sigma_{i} \in S, \quad \text { and } \\
f(\alpha) & =0, \quad \alpha \neq \sigma_{i} r, \quad \sigma_{i} \in S, \quad r \in D .
\end{aligned}
$$

Let $k:=h(f+g)-h g$ where $h$ is defined by $h\left[\begin{array}{l}1 \\ 0\end{array}\right]=\left[\begin{array}{l}0 \\ 0\end{array}\right], h\left[\begin{array}{l}x \\ y\end{array}\right]=\left[\begin{array}{l}x \\ x\end{array}\right]$ if $y \neq 0$ and $g$ is defined recursively as follows.

Since $D$ is not a field, $D$ does not satisfy the descending chain condition for right ideals so we let $A_{1}, A_{2}, \ldots$, be a strictly descending chain with the property $\cap A_{i}=\{0\}$. (For example one might take any non-unit $a \in D$ and let $A_{i}=a^{i} D$.) Note that for every $x \in A_{1}$ there is a unique integer $\varphi(x)$ such that $x \in A_{\varphi(x)} \backslash A_{\varphi(x)+1}$. Choose $a_{1} a_{2}, \ldots$, such that $a_{i} \in A_{i} \backslash A_{i+1}$. We define

$$
g\left(\sigma_{1}\right):=\left[\begin{array}{c}
\left(a_{1}-1\right) x_{1} \\
0
\end{array}\right]
$$

and set $j_{0}=0, j_{1}=\varphi\left(a_{1} x_{1}\right)$. Now suppose $g\left(\sigma_{i}\right)$ and $j_{i}, i=1,2, \ldots, n$, have been defined. Then

$$
g\left(\sigma_{n+1}\right):=\left[\begin{array}{c}
\left(a_{j_{n}+1}-1\right) x_{n+1} \\
0
\end{array}\right] \text { and } j_{n+1}:=\varphi\left(a_{j_{n}+1} x_{n+1}\right) .
$$


From this we obtain

$$
k\left(\sigma_{n+1}\right)= \begin{cases}{\left[\begin{array}{l}
a_{1} x_{1} \\
a_{1} x_{1}
\end{array}\right]} & \text { if } n=0, \\
{\left[\begin{array}{ll}
a_{j_{n}+1} x_{n+1} \\
a_{j_{n}+1} x_{n+1}
\end{array}\right]} & \text { if } n>0 .\end{cases}
$$

Thus we have constructed a function $k$ in the left ideal generated by $f$ such that for $\sigma, \tau \in S$, if $\sigma \neq \tau$ then $k(\sigma) \neq k(\tau)$ and

$$
k\left(\sigma_{i}\right)=\left[\begin{array}{l}
z_{i} \\
z_{i}
\end{array}\right] .
$$

Define

$$
\delta_{i}:=\left[\begin{array}{c}
z_{i}+1 \\
z_{i}
\end{array}\right], \quad i=1,2, \ldots
$$

From Example 3.1, the $\delta_{i}$ are generators. We define $g_{1}, h_{1}$ in $N$ by $g_{1}\left(\delta_{i}\right)=\left[\begin{array}{l}1 \\ 0\end{array}\right], g_{1}(x)=0$ for $x \neq \delta_{i} r, r \in D, i=1,2, \ldots$, and $h_{1}\left(\delta_{i}\right)=\sigma_{i}$, $h_{1}(x)=0, x \neq \delta_{i} s, s \in D, i=1,2, \ldots$. Now let $l:=h_{1}\left(k+g_{1}\right)-h_{1} g_{1}$ which is in the left ideal generated by $f$. For each $\sigma_{i} \in S$,

$$
l\left(\sigma_{i}\right)=h_{1}\left(\left[\begin{array}{l}
z_{i} \\
z_{i}
\end{array}\right]+\left[\begin{array}{l}
1 \\
0
\end{array}\right]\right)=h_{1}\left(\delta_{i}\right)=\sigma_{i}
$$

This is our desired function.

Now let $l_{0}$ be defined by $l_{0}\left(\sigma_{i}\right)=0, \sigma_{i} \in S, l_{0}(x)=x-l(x), x \neq \sigma_{i} r$, $r \in D, \sigma_{i} \in S$. Then $l_{0}+l$ is in the left ideal generated by $L(\mathscr{F}) \cup\{f\}$. But $l_{0}+l=\mathrm{id}$. This establishes the next result. We note also that a similar result has been established by Fuchs in [1].

Theorem 3.9. Let $N:=M_{D}\left(D^{2}\right)$ where $D$ is an integral domain, not necessarily commutative, and $D$ is not a field. Let $H$ denote the set of distinct generators in $D^{2}$ and let $\mathscr{F}$ be an ultrafilter on $H$ with the property that for each $A \in \mathscr{F}$, there exists a countable set $B, B \subseteq A$, such that $B \in \mathscr{F}$. Then $L(\mathscr{F})=\{f \in N \mid f(A)=\{0\}$ for some $A \in \mathscr{F}\}$ is a maximal left ideal of $N$.

The case in which $D$ is a field will be discussed later. We now turn to a characterization of the two-sided (or invariant) subgroups of $M_{D}\left(D^{2}\right)$.

We take $D$ to be a PID and as usual we let $N:=M_{D}\left(D^{2}\right)$. Let $A$ be any ideal of $D$ and define $A^{+}=\left\{f \in N \mid f(\alpha) \in A^{2}\right.$, for all $\left.\alpha \in D^{2}\right\}$. We note that $A^{+}$is a subgroup of $N$ and for any $h, k \in N, h f k \in A^{+}$, since $A$ is principal. Thus $A^{+}$is an $(N, N)$-subgroup, that is, an invariant subgroup of $N$. As above, we let $H$ denote a set of generators, one chosen from each equivalence class. 
LemMA 3.10. Let $D$ be a PID, $A, B$ ideals of $D$. If $A \neq B$ then $A^{+} \neq$ $B^{+}$.

Proof. Suppose $a \in A \backslash B$. Let $\alpha \in H$ and define $f(\alpha)=\left[\begin{array}{l}a \\ a\end{array}\right]$ and $f(\beta)=\left[\begin{array}{l}0 \\ 0\end{array}\right]$ for each $\beta \in H, \beta \neq \alpha$. Then $f$ is a function in $N$ and $f \in A^{+} \backslash B^{+}$.

LEMMA 3.11. Every invariant subgroup $T$ of $N$ is of the form $T=A^{+}$ for some ideal $A$ of $D$.

Proof. If $T=\{0\}$ the result is clear so we take $T \neq\{0\}$ and let

$$
T_{+}:=\left\{a \in D \mid \text { for some } \gamma \in D^{2} \text { and } f \in T, f(\gamma)=\left[\begin{array}{l}
a \\
0
\end{array}\right]\right\} \text {. }
$$

Clearly if $a \in T_{+}$then $a r \in T_{+}$for each $r \in D$. Suppose $a, b \in T_{+}$. Then there exist $f_{1}, f_{2} \in T, \gamma_{1}, \gamma_{2} \in D^{2}$ with $f_{1}\left(\gamma_{1}\right)=\left[\begin{array}{l}a \\ 0\end{array}\right], f_{2}\left(\gamma_{2}\right)=\left[\begin{array}{l}b \\ 0\end{array}\right]$. If $\alpha$ is any generator then there exist $h, k \in N$ with $h(\alpha)=\gamma_{1}, k(\alpha)=\gamma_{2}$. Hence $\left(f_{1} h+f_{2} k\right) \alpha=\left[\begin{array}{c}a+b \\ 0\end{array}\right]$. Therefore $T_{+}$is an ideal of $D$, say $T_{+}=(m)$. We next show $T=\left(T_{+}\right)^{+}$. We have $T \subseteq\left(T_{+}\right)^{+}$so we turn to the reverse inclusion.

From Lemma 2.6, $T \cap \mathbf{M}_{2}(D) \neq\{0\}$. But then from the definition of $T_{+}$we must have $T \cap \mathbf{M}_{2}(D)=\mathbf{M}_{2}\left(T_{+}\right)$. Let $f \in\left(T_{+}\right)^{+}$. For each $\alpha \in$ $H, f(\alpha) \in\left(T_{+}\right)^{2}$, say $f(\alpha)=\left[\begin{array}{c}m r(\alpha) \\ m s(\alpha)\end{array}\right], r(\alpha), s(\alpha) \in D$. Define $f^{\prime}$ as the function obtained from $f, f^{\prime}(\alpha):=\left[\begin{array}{l}r(\alpha) \\ s(\alpha)\end{array}\right], \alpha \in H$. Clearly $f^{\prime} \in N$. From the definition of $T_{+}$we know there exist $g \in T,\left[\begin{array}{l}c \\ d\end{array}\right] \in D^{2}$, with $g\left[\begin{array}{l}c \\ d\end{array}\right]=\left[\begin{array}{l}m \\ 0\end{array}\right]$. As in Lemma 2.6, $g\left[\begin{array}{ll}0 & c \\ 0 & d\end{array}\right]=\left[\begin{array}{ll}0 & m \\ 0 & 0\end{array}\right]$, so $\left[\begin{array}{ll}0 & m \\ 0 & 0\end{array}\right] \in T$. But then $\left[\begin{array}{cc}m & 0 \\ 0 & m\end{array}\right] \in T$, and consequently $f=\left[\begin{array}{cc}m & 0 \\ 0 & m\end{array}\right] f^{\prime} \in T$. Therefore $\left(T_{+}\right)^{+} \subseteq T$ and we have the result.

From the above proof we obtain the next result.

Corollary 3.12. Let $T_{1}$ and $T_{2}$ be invariant subgroups of $M_{D}\left(D^{2}\right), D$ a PID. If $T_{1} \neq T_{2}$ then $\left(T_{1}\right)_{+} \neq\left(T_{2}\right)_{+}$.

Corollary 3.13. Let $D$ be a PID. If $A$ is an ideal of $D$ then $\left(A^{+}\right)_{+}=A$.

Proof. We know $\left(A^{+}\right)_{+}$is an ideal of $D$, say $B:=\left(A^{+}\right)_{+}$. As in the above proof, $B^{+}=\left(\left(A^{+}\right)_{+}\right)^{+}=A^{+}$. But then from Lemma 3.10, $A=B$.

From the above results it is straightforward to verify that the map $A \rightarrow$ $A^{+}, A$ an ideal of $D$, is an order preserving bijection between the lattice of ideals of $D$ and the lattice of invariant subgroups of $N$. We summarize these results in the following theorem. 
THEOREM 3.14. Let $D$ be $a$ PID and $N:=M_{D}\left(D^{2}\right)$. There is a lattice isomorphism between the lattice of ideals of $D$ and the lattice of invariant subgroups of $N$.

We conclude this section and the paper with a discussion of the case in which $N:=M_{F}\left(F^{2}\right), F$ a field. Thus $F^{*}$ is a group of fixed point free automorphisms acting on a group $F^{2}$ and some of our results follow from the general theory of such actions.

From Theorem 2.8, $M_{F}\left(F^{2}\right)$ is a simple near-ring. We now show that for left ideals one obtains a nice relationship with filters. Thus we let $F$ denote a field and let $H:=\left\{\sigma_{\alpha}:=\left[\begin{array}{l}a \\ 1\end{array}\right] \mid a \in F\right\} \cup\left\{\sigma_{\infty}:=\left[\begin{array}{l}1 \\ 0\end{array}\right]\right\}$, a set of generators, one from each equivalence class.

LEMMA 3.15. Let $S \subseteq H$ be a non-empty set of generators. If $f \in M_{F}\left(F^{2}\right)$ is non-zero on $S$ and zero off $S$, then there exists a function $k$ in the left ideal generated by $f$ such that $k(\sigma)=\sigma, \sigma \in S$, and $k(\sigma)=\left[\begin{array}{l}0 \\ 0\end{array}\right], \sigma \in H \backslash S$.

Proof. Let $S=\left\{\sigma_{i}\right\}, i \in I$. Without loss of generality we take

$$
f\left(\sigma_{i}\right)=\left[\begin{array}{l}
x_{i} \\
x_{i}
\end{array}\right] .
$$

Let $T:=\left\{\delta_{i}\right\}, i \in I$, be a subset of $H$ such that $\sigma_{\infty} \notin T$. We take

$$
\delta_{i}=\left[\begin{array}{c}
a_{i} \\
1
\end{array}\right], \quad i \in I
$$

Define $h, g \in M_{F}\left(F^{2}\right)$ by $h\left(\sigma_{\infty}\right)=\left[\begin{array}{l}0 \\ 0\end{array}\right], h\left(\delta_{i}\right)=\delta_{i} x_{i}^{-1}, i \in I, h(\alpha)=\left[\begin{array}{l}0 \\ 0\end{array}\right]$ otherwise and

$$
g\left(\sigma_{i}\right)=\left[\begin{array}{c}
\left(a_{i}-1\right) x_{i} \\
0
\end{array}\right], \quad g(\alpha)=\left[\begin{array}{l}
0 \\
0
\end{array}\right] \quad \text { otherwise } .
$$

Then $k:=h(f+g)-h g$ is in the left ideal generated by $f$ and

$$
k\left(\sigma_{i}\right)=h\left(\left[\begin{array}{l}
x_{i} \\
x_{i}
\end{array}\right]+\left[\begin{array}{c}
\left(a_{i}-1\right) x_{i} \\
0
\end{array}\right]\right)-h\left[\begin{array}{c}
\left(a_{i}-1\right) x_{i} \\
0
\end{array}\right]=h\left[\begin{array}{c}
a_{i} \\
1
\end{array}\right] x_{i}=\sigma_{i}
$$

and $k$ is zero off $S$, and hence we have the result.

For any $f \in M_{F}\left(F^{2}\right)$ we define the zero set $Z_{f}$ of $f$ by $Z_{f}:=\{\alpha \in$ $\left.H \mid f(\alpha)=\left[\begin{array}{l}0 \\ 0\end{array}\right]\right\}$.

CoROllaRy 3.16. If $I$ is a proper left ideal of $M_{F}\left(F^{2}\right)$ and $f \in I$ then $Z_{f}$ $\neq \varnothing$. 
LEMMA 3.17. Let $I$ be a proper left ideal of $M_{F}\left(F^{2}\right), 0 \neq f \in I$ and let $\alpha \in F^{2}$. There exists a function $k \in I$ such that

$$
k(\sigma)= \begin{cases}0, & \sigma \in Z_{f}, \\ \alpha, & \sigma \in H \backslash Z_{f} .\end{cases}
$$

Proof. From Lemma 3.15, we may assume without loss of generality that $f$ is the identity on $Z_{f}$. Let $h$ be defined by $h(\sigma)=\alpha$ for each $\alpha \in H$. Then $k:=h f$ is the desired function.

LEMMA 3.18. Let $I$ be a proper left ideal of $M_{F}\left(F^{2}\right)$ and set $\mathscr{F}(I):=$ $\left\{Z_{f} \mid f \in I\right\}$. Then $\mathscr{F}(I)$ is a filter on $H$.

Proof. Clearly $\varnothing \notin \mathscr{F}(I)$. Suppose $Z_{f}, Z_{g} \in \mathscr{F}(I)$. Let $\alpha, \beta \in F^{2}$ be such that $\alpha \neq\left[\begin{array}{l}0 \\ 0\end{array}\right] \neq \beta$ and $\alpha+\beta \neq\left[\begin{array}{l}0 \\ 0\end{array}\right]$. From Lemma 3.17, there exist functions $f^{\prime}, g^{\prime} \in M_{F}\left(F^{2}\right)$ such that $f^{\prime}(\sigma)=\alpha$ off $Z_{f}, g^{\prime}(\sigma)=\beta$ off $Z_{g}$ and $Z_{f^{\prime}}=Z_{f}, Z_{g^{\prime}}=Z_{g}$. Then $Z_{f} \cap Z_{g}=Z_{f^{\prime}+g^{\prime}}$. But $f^{\prime}+g^{\prime} \in I$ so $Z_{f} \cap Z_{g} \in \mathscr{F}(I)$. Finally, let $Z_{f} \subseteq S$. Then $f \in I$ is zero on $Z_{f}$, non-zero off $Z_{f}$. From Lemma 3.15 , there exists $k \in I$ such that $k$ is the identity off $Z_{f}$ and zero on $Z_{f}$. Define $h \in M_{F}\left(F^{2}\right)$ by $h(\sigma)=\sigma, \sigma \in H \backslash S$, $h(\sigma)=\left[\begin{array}{l}0 \\ 0\end{array}\right], \sigma \in S$. Then $h k \in I$ and $Z_{h k}=S$, so $S \in \mathscr{F}(I)$.

LEMMA 3.19. Let $I$ be a proper left ideal of $M_{F}\left(F^{2}\right)$. Then $I^{*}:=\{f \in$ $\left.M_{F}\left(F^{2}\right) \mid Z_{f} \in \mathscr{F}(I)\right\}=I$.

Proof. We have $I \subseteq I^{*}$. For the reverse inclusion, let $f^{*} \in I^{*}$. Since $Z_{f^{*}} \in \mathscr{F}(I)$, there exists a function $g \in I$ with $Z_{g}=Z_{f^{*}}$. As in the proof of Lemma 3.17 we may take $g(\sigma)=\sigma, \sigma \in H \backslash Z_{f^{*}}$. But then $f^{*}=f^{*} g \in I$.

Let $\mathscr{F}$ be a filter on $H$ and define $I(\mathscr{F}):=\left\{f \in M_{F}\left(F^{2}\right) \mid Z_{f} \in \mathscr{F}\right\}$. Using the properties of $\mathscr{F}$ it is straightforward to verify that $I(\mathscr{F})$ is a left ideal of $M_{F}\left(F^{2}\right)$. Our final theorem follows from this remark and the above sequence of lemmas. Recall that a filter $\mathscr{F}$ on $H$ is said to be a minimal filter if $\mathscr{F}$ consists only of $H$ and just one other set $S$ such that $H \backslash S$ is a singleton.

THEOREM 3.20. There is an order preserving bijection between the proper left ideals of $M_{F}\left(F^{2}\right), F$ a field, and filters on $H$. An ultrafilter corresponds to a maximal left ideal and a minimal filter corresponds to a minimal left ideal. 


\section{Acknowledgement}

This paper was written while the first author was visiting the Department of Mathematics at the University of Stellenbosch in 1988. He wishes to express his deep appreciation for the hospitality and financial assistance provided by the University of Stellenbosch in support of this visit.

\section{References}

[1] P. Fuchs, Ultraproducts of $\Omega$-groups, (Ph.D.-thesis, Linz, 1985).

[2] C. J. Maxson and K. C. Smith, 'Simple near-ring centralizers of finite rings', Proc. Amer. Math. Soc.75 (1979), 8-12.

[3] C. J. Maxson and K. C. Smith, 'Centralizer near-rings that are endomorphism rings', Proc. Amer. Math. Soc. 80 (1980), 189-195.

[4] J. D. P. Meldrum, Near-rings and their links with groups, (Pitman Research Notes Series, No 134, 1985).

[5] G. Pilz, Near-rings, (North-Holland, American Elsevier, Amsterdam, 1983).

Department of Mathematics

Texas A \& M University

College Station

Texas 77843

U.S.A.
Department of Mathematics

Stellenbosch University

Stellenbosch, 7600

South Africa 